\title{
Granulomatosis With Polyangiitis Presenting as Prostatitis: An Uncommon Scenario
}

\author{
Joseph Chan ${ }^{\mathrm{a}, \mathrm{b}}$, Mauro Braun ${ }^{\mathrm{a}}$, Hossam Elzawawy ${ }^{\mathrm{a}}$, Mariana Berho ${ }^{\mathrm{a}}$, Jacobo Kirsch ${ }^{\mathrm{a}}$
}

\begin{abstract}
Granulomatosis with Polyangiitis (GPA) formerly known as Wegener's Granulomatosis is an ANCA associated small vessel vasculitis that commonly involves the pulmonary and renal system. The lower urogenital tract is rarely affected. We present a patient who developed prostatitis initially and later manifested itself with multisystemic involvement from GPA.
\end{abstract}

Keywords: Granulomatosis with Polyangitis; Wegener's granulomatosis; Prostatitis

\section{Introduction}

Granulomatosis with Polyangiitis (GPA) formerly known as Wegener's Granulomatosis is an ANCA associated small vessel vasculitis that can affect multiple organ systems. Most commonly it involves the upper respiratory tract causing epistaxis and nasal ulceration. Lower respiratory tract involvement occurs in $80 \%$ of cases and may present with hemoptysis and radiologic findings of pulmonary nodules or infiltrates. Renal involvement is also quite common (46\%) [1]. However, the lower urogenital tract is rarely affected. Moreover, initial manifestation of prostatic symptoms is rare $(2.3-7.4 \%)[1,2]$. We present a patient who developed prostatitis that later manifested itself with multi-systemic involvement from GPA.

\section{Case Report}

A 48 year old Caucasian male initially presented to the emer-

Manuscript accepted for publication June 12, 2012

${ }^{a}$ Department of Nephrology and Hypertension, Cleveland Clinic Florida, 2950 Cleveland Clinic Blvd, Weston, Florida 33331, USA

${ }^{\mathrm{b}}$ Corresponding author: Joseph Chan. Email: chanj@ccf.org

doi:10.4021/wjnu18w gency department with hematuria, dysuria and treated with oral antibiotics for urinary tract infection (UTI). He returned days later with urinary retention fever and chills and was admitted for IV antibiotics. CBC was significant for anemia, leukocytosis and thrombocytosis. Creatinine and electrolytes were normal. Urinalysis was notable for pyuria. A distended bladder and an enlarged prostate were seen on abdominal CT scan. He required insertion of a Foley catheter by cystoscopy, which drained large amount of urine. He was discharged on Ciprofloxacin. On follow up with his urologist, he had transurethral resection of the prostate (TURP) and a prostate biopsy revealed severe necrotizing, pallisading granulomatous inflammation with giant cell formation (Fig. 1) and was transitioned to trimethoprim/sulfamethoxazole.

One week later he developed polyarthralgias and swelling of feet and ankles with modest improvement with oral steroids and tramadol. This was followed by hyperhydrosis of the right side of the face. An MRI of the head and neck was unremarkable except for an incidental finding of a 5.6 $\mathrm{cm}$ cavitary lesion on the right apex (Fig. 2). The patient denied any dyspnea, cough or hemoptysis. CT scan of his chest showed bilateral lung nodules and a cavitary mass in the right lung apex measures $5.8 \times 4.4 \mathrm{~cm}$. Subsequently he had eruption of palpable purpuric lesions on his lower extremities, which prompted him to go to the emergency department.

On physical exam he was afebrile and normotensive. Nasal turbinates and oral mucosa were clear. Auscultation of lungs showed vesicular breath sounds. Palpable purpuric lesion noted on both arms and lower extremities. CBC was significant for anemia. ESR and CRP were elevated $116 \mathrm{~mm} /$ $\mathrm{hr}, 68 \mathrm{mg} / \mathrm{L}$ respectively. He was in non oliguric renal failure with a creatinine of $3.2 \mathrm{mg} / \mathrm{dL}$ and non nephrotic range proteinuria. Urine electrolytes were not consistent with volume depletion, and renal ultrasound showed normal sized kidney bilaterally without hydronephrosis. Serologic workup for glomerulonephritis was done and C-ANCA and Anti PR3 antibodies were positive. Granulomatosis with polyangiitis was diagnosed after a renal biopsy revealed diffuse crescentic and necrotizing glomerulonephritis (Fig. 3). The skin biopsy showed necrotizing granulomas of small and medium sized arteries. The right upper lung cavity was biopsied and 


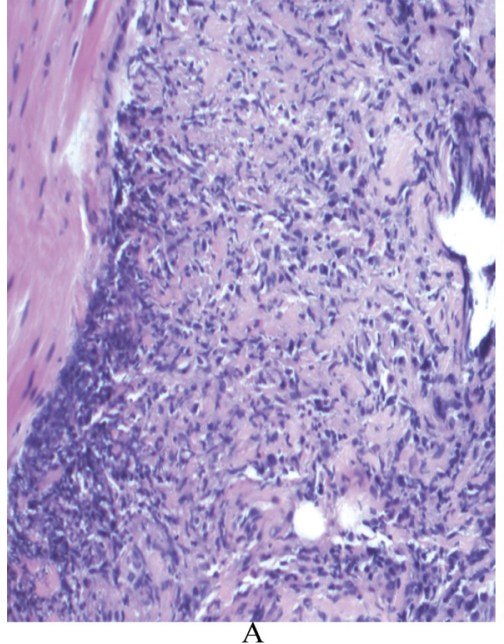

A

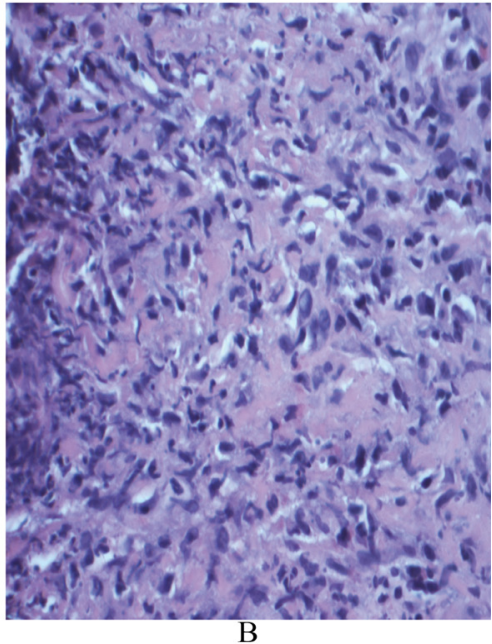

B

Figure 1. A: Scanning magnification of the prostate tissue shows extensive replacement by granulomatous inflammation (x 1000; $\mathrm{H}$ and E); $\mathrm{B}$ : High power view demonstrates small foci of necrosis within the granulomas ( $\mathrm{x} 4000 ; \mathrm{H}$ and $\mathrm{E})$.

cultures grew both alpha and beta hemolytic streptococci without acid fast bacilli. Cytology did not show evidence of malignancy. The lung biopsy, however, was not adequate for pathology. Transesophageal echocardiogram showed no evidence of infective endocarditis. High dose prednisone and oral cytoxan was commenced while the patient was receiving IV antibiotics. However despite immunosuppressive measures, he progressed to oliguric renal failure requiring dialysis alternated with plasmapharesis. His dermatological and arthritic complaints abated. He had absence of hemoptysis or respiratory complaints. The patient's renal function did not return to baseline and has been dialysis dependent since.

\section{Discussion}

Granulomatosis with Polyangitis is an anti cytoplasmic antibody (ANCA) associated systemic vasculitis which can involve multiple organ systems. Etiology is unknown but development of anti cytoplasmic antibodies can trigger an autoimmune response. Infection also has been linked to its pathogenesis [3-5]. The mechanism of which is not completely understood. Peak incidence occurs in the fifth and sixth decade of life. It can affect the respiratory tract, kidneys and less commonly, the urogenital tract. Diagnosis relies on serology (ANCA positivity) particularly autoantibodies to proteinase 3 (PR3-ANCA) as well as tissue biopsy. PR3ANCA has a sensitivity of $70-80 \%$ and specificity of $98 \%$ [6]. Mainstay of treatment involves immunosuppressive medications such as systemic steroids and cyclophophamide. Most recently, rituximab has been used to induce remission

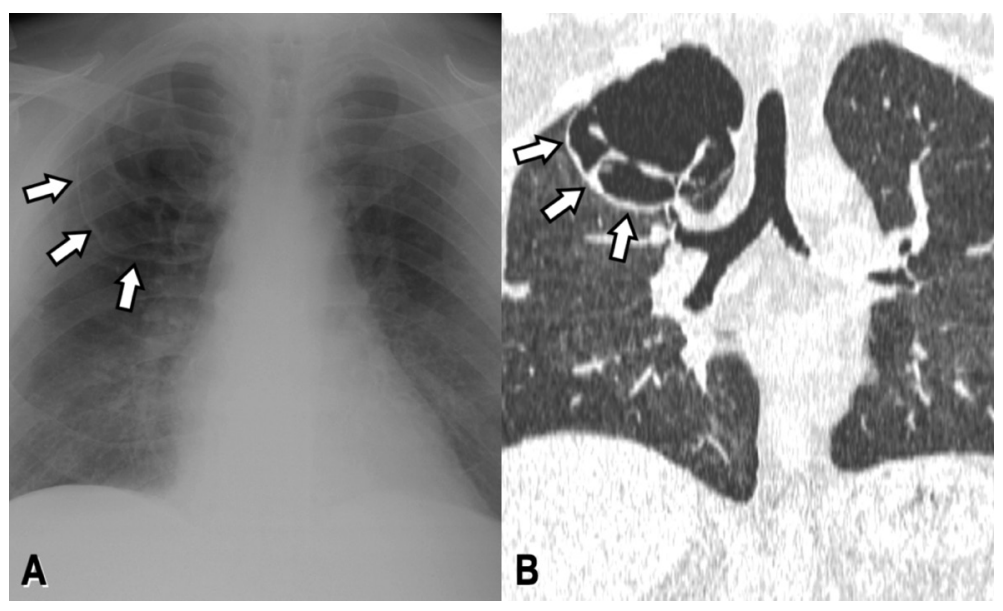

Figure 2. PA chest $x$-ray $(A)$ and coronal $C T$ reconstruction $(B)$ reveal a large septated cystic lesion replacing most of the superior segment of the right upper lobe (white arrows). 


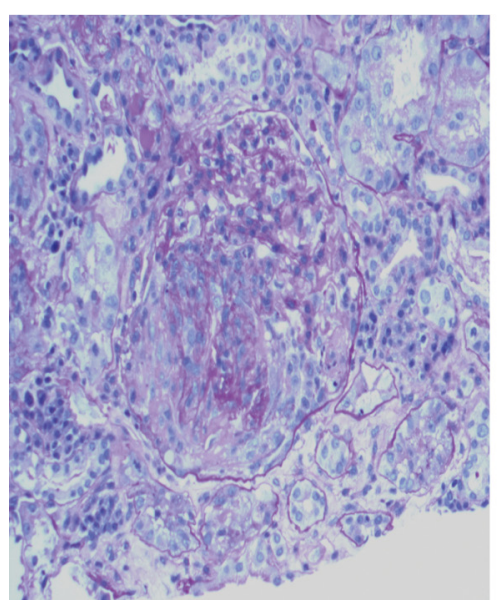

A

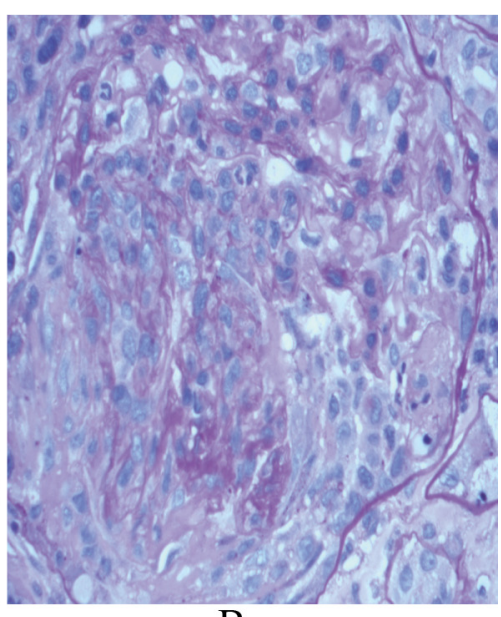

B

Figure 3. A: PAS stain reveals crescent formation within the glomerulus with central collapse of the capillary loops (x 2000; PAS stain); B: Higher magnification shows segmental areas of fibrinoid necrosis (x 4000; PAS stain).

in ANCA associated vasculitis and may have less adverse effects when compared with cyclophosphamide [7]. Respiratory support, plasmapharesis and dialysis are often times utilized in severe cases of hemoptysis and continued decline in renal function despite immunosuppressive therapy.

The initial urogenital symptom of our patient is an uncommon presentation for GPA. Urogential involvement has been said to occur in 2.3-7.4\% of patients with GPA. When GPA involves the urogenital tract, the prostate is most commonly affected but can also affect epidydimis, penis, testes, and seminal vesicles. His biopsy, consistent with prostatitis, was initially attributed to an infectious cause. Other etiologies for granulomatous prostatitis include non infectious and can be related to urological procedure such as TURP, prostatectomy, needle biopsy and instillation of BCG into the bladder [8]. A literature review by Middleton et al found 18 case reports of prostatic symptoms due to GPA. In this review, only $7 / 18$ presented initially with prostatic complaints. Symptoms vary from dysuria, gross hematuria or urinary retention. His urinary complaints abated after treatment with trimetroprim/sulfamethoxazole. There is confounding evidence that this sulfa based antibiotic has a role in the prevention of relapse in GPA. Lung involvement occurs in $80 \%$ of GPA cases making it the most common organ affected in GPA. Symptoms vary from minimal to life threatening [9]. Another interesting finding in our case was the massive cavitary lesion seen on radiography. His symptoms however did not correlate well with these CT findings as he did not have dypnea, hemoptysis, cough or pleurisy. Hemoptysis is the most common reason for admission in intensive care unit in patients with vasculitis [10]. Hemoptysis is more common in patients with diffuse bilateral infiltrates, which can be due to alveolar filling. Although a repeat CT scan showed new bilateral infiltrates, bronchoscopy in our patient did not detect active bleeding.

The lung biopsy identified polymicrobial organisms. Infections have been linked to a causative role in intiating the disease process in GPA. In a review by Pagnoux et al, vasculitis following bacterial infections results from direct invasion of endothelial cells. Only $50 \%$ will have positive blood cultures. Of note, blood cultures in our patient did not grow any organisms. Autoimmune T- and/or B-cell reactions triggered by microbial antigens may perhaps induce disease in susceptible patients by epitope mimicry or superantigen induced T-cell activation [11]. It is unclear in our patient if infection was the culprit initiating GPA. The cavitary lung lesion was an incidental finding and it was not known if these lesions were present prior to the onset of prostatitis. It is plausible to think that this infectious cavitary lesion was a result of aspiration or an effect rather than the cause of the GPA. In our case, proper coordination of treatment plan with an infectious disease expert proved to be very helpful. Prompt antibiotic therapy is warranted if infection is suspected. Plasma exchange (PE) has been used to reduce disease progression [12]. A proposed mechanism is through elimination of immune-complexes. Due to the progressive nature of his renal failure despite cyclophosphamide and high dose steroids, PE was done on an attempt to induce remission. His pulmonary lesions improved but renal function continued to deteriorate. This also has been the case in Bethoux's study in which PE was used in adjunct to steroids and cyclophosphamide to treat alveolar hemorrhage in a patient with renal failure from GPA. Although plasma exchange has not proven to decrease mortality in GPA, it is conceivable that it may decrease the severity of the disease.

GPA can affect multiple organs and genitorurinary complaints may be the intial presentation. Prompt diagnosis and initiation of treatment has a pivotal role in inducing remis- 
sion. Urologists may be the initial caregivers for these rare cases and should consider autoimmune vasculitis as a cause of prostatitis especially when presentation is atypical. Proper use of antibiotics in cases where a source of infection is identified prior to the initiation of immunosuppressive therapy is prudent. Although plasma exchange has been shown to be of use in severe cases, renal disease can progress despite therapy.

\section{Conflicts of Interest}

The authors in regard to this work declared No conflict of interest.

\section{References}

1. Stillwell TJ, DeRemee RA, McDonald TJ, Weiland LH, Engen DE. Prostatic involvement in Wegener's granulomatosis. J Urol. 1987;138(5):1251-1253.

2. Walton EW. Giant-cell granuloma of the respiratory tract (Wegener's granulomatosis). Br Med J. 1958;2(5091):265-270.

3. George J, Levy Y, Kallenberg CG, Shoenfeld Y. Infections and Wegener's granulomatosis--a cause and effect relationship? QJM. 1997;90(5):367-373.

4. See CQ, Jaffe HA, Schraufnagel DE. Dyspnea and he- moptysis develop in a young man with prostatitis. Chest. 2005;128(5):3625-3628.

5. Pagnoux C, Cohen P, Guillevin L. Vasculitides secondary to infections. Clin Exp Rheumatol. 2006;24(2 Suppl 41):S71-81.

6. Middleton G, Karp D, Lee E, Cush J. Wegener's granulomatosis presenting as lower back pain with prostatitis and ureteral obstruction. J Rheumatol. 1994;21(3):566569.

7. Stone JH, Merkel PA, Spiera R, Seo P, Langford CA, Hoffman GS, Kallenberg CG, et al. Rituximab versus cyclophosphamide for ANCA-associated vasculitis. N Engl J Med. 2010;363(3):221-232.

8. Uzoh CC, Uff JS, Okeke AA. Granulomatous prostatitis. BJU Int. 2007;99(3):510-512.

9. Gascon A, Cobeta JC, Iglesias E. Is Gitelman's syndrome an acquired disease? Br J Rheumatol. 1998;37(7):794.

10. Khan SA, Subla MR, Behl D, Specks U, Afessa B. Outcome of patients with small-vessel vasculitis admitted to a medical ICU. Chest. 2007;131(4):972-976.

11. Berthoux E, Padilla M, Chavez L, Colombe B, Bosseray A, Massot C. Unusual evolution in Wegener's granulomatosis: recovery of pulmonary involvement while renal disease progressed to end-stage. Ren Fail. 2011;33(10):1032-1036.

12. Guillevin L, Pagnoux C. Indications of plasma exchanges for systemic vasculitides. Ther Apher Dial. 2003;7(2):155-160. 